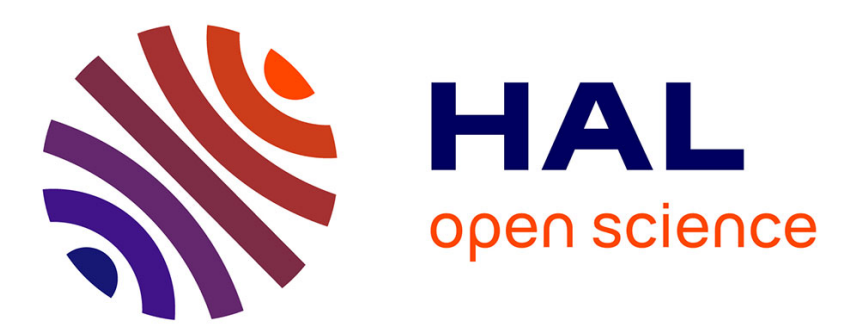

\title{
Trahisons et idées de trahison sous les princes angevins et normands (1050-1150) \\ Bruno Lemesle
}

\section{To cite this version:}

Bruno Lemesle. Trahisons et idées de trahison sous les princes angevins et normands (1050-1150). La trahison au Moyen Age. De la monstruosité au crime politique (Ve-XVe siècle), Jun 2008, Lyon, France. pp.229-238. halshs-00493954

\section{HAL Id: halshs-00493954 https://shs.hal.science/halshs-00493954}

Submitted on 9 Jul 2010

HAL is a multi-disciplinary open access archive for the deposit and dissemination of scientific research documents, whether they are published or not. The documents may come from teaching and research institutions in France or abroad, or from public or private research centers.
L'archive ouverte pluridisciplinaire HAL, est destinée au dépôt et à la diffusion de documents scientifiques de niveau recherche, publiés ou non, émanant des établissements d'enseignement et de recherche français ou étrangers, des laboratoires publics ou privés. 


\title{
TRAHISONS ET IDEES DE TRAHISON SOUS LES PRINCES ANGEVINS ET NORMANDS (1050-1150)
}

\author{
Bruno Lemesle
}

Voici comment la chronique des comtes d'Anjou, rédigée dans la première moitié du $\mathrm{XII}^{\mathrm{e}}$ s., évoque les événements de 1067 qui ont conduit à l'éviction du comte Geoffroy le Barbu au profit de son frère Foulque :

«Foulque, par sa fourberie, engageant une lutte démesurée contre son frère, précipita le comté tout entier dans le désordre. Partout, les barons se dressèrent les uns contre les autres, soutenant tantôt le Barbu, tantôt Foulque. Entre eux, des trahisons multiples furent commises. En celle d'Angers, en l'an 1066 de l'Incarnation du Seigneur, les traîtres périrent ${ }^{1}$. »

Comme on le voit, l'auteur du récit évoque les trahisons des barons comme des conséquences du coup de force perpétré par le frère du comte ${ }^{2}$. S'il évite de nommer « trahison » l'action de Foulque proprement dite, c'est parce que celui-ci a gouverné le comté d'Anjou jusqu'à sa mort en 1109 et que l'auteur appartient à un milieu pro-angevin. Le moine normand Orderic Vital, en revanche, ne s'est pas senti tenu à pareille précaution, choisissant pour sa part de souligner l'illégitimité du renversement : "Foulque, écrit-il, surnommé le Réchin, se rebella contre son frère et seigneur Geoffroy, le captura par trahison et le tint enfermé dans la prison du château de Chinon pendant plus de trente ans ${ }^{3}$. » Les mêmes termes sont repris lorsqu'il évoque la demande de libération de Geoffroy par le pape Urbain II en $1096^{4}$. Nous remarquons qu'Orderic Vital associe explicitement les termes de trahison et de rébellion : cette association est fréquente chez lui. Utilisée comme fil conducteur, elle permet d'évaluer les pratiques et le concept de trahison aux XI ${ }^{\mathrm{e}}$ et XII ${ }^{\mathrm{e}}$ s. En 1981, S. Cuttler, dans une étude portant sur la fin du Moyen Âge, rappelle que le fondement juridique de la trahison est romain. Il estime aussi qu'entre le $\mathrm{VI}^{\mathrm{e}}$ et le $\mathrm{XI}^{\mathrm{e}}$ s., l'influence du droit romain étant devenue négligeable, la trahison ne se manifesterait principalement que sous la forme, atténuée, de l'infidélité. Il s'agit de l'infidélité du vassal à l'égard de son seigneur. C'est en

\footnotetext{
1 « Fulco subdolus fratrem suum nimium cepit inpugnare et consulatum totum turbare; et tunc totius comitatus barones unus in alium ceperunt insurgere, nunc Barbato, nunc Fulconi favere ; et tunc proditiones multe inter eos exorte sunt. Quo tempore fit proditio aput Andegavem, anno Verbi incarnati M. ${ }^{\mathrm{mo}} \mathrm{LX}^{\mathrm{mo}} \mathrm{VI}^{\mathrm{to}}{ }^{\mathrm{t}}$; proditores perimuntur », "Chronica de gestis consulum andegavorum », Chroniques des comtes d'Anjou et des seigneurs d'Amboise, éd. L. Halphen et R. Poupardin, Paris, 1913, p. 63. C'est le 4 avril 1067 que Foulque a capturé son frère, à Angers, grâce à la trahison de ses partisans, L. Halphen, Le comté d'Anjou au XI $I^{e}$ s., Genève, 1974 [1906].

${ }^{2}$ Les traîtres victimes de l'émeute provoquée par ces événements, partisans de Foulque, sont trois seigneurs de château, Geoffroy de Preuilly, Renaud de Château-Gontier, Giraud de Montreuil-Bellay, ainsi qu'un prévôt du comte, Robert. Un autre partisan de Foulque est le seigneur de Sablé, Robert, dans le château duquel Geoffroy le Barbu est emprisonné, «Annales de Vendôme et de Renaud », Recueil d'annales angevines et vendômoises, éd. L. Halphen, Paris, 1903, p. 64 et 87 ; Cartularium monasterii Beatae Mariae Andegavensis, Archives d'Anjou, éd. P. Marchegay, Angers, t. 3, 1854, $\mathrm{n}^{\circ} 176$.

3 «Fulco cognomento Richinus contra Goisfredum fratrem dominumque suum rebellauit, eumque per proditionem comprehendit, et plus quam XXX annis in carcere Chinonis castri clausum tenuit », Orderic Vital, Historia ecclesiastica, éd. et trad. M. Chibnall, The Ecclesiastical History of Orderic Vitalis, vol. II, liv. IV, Oxford, 1969, p. 304-306. Moine à l'abbaye de Saint-Évroul en Normandie, Orderic Vital a composé son Histoire ecclésiastique vraisemblablement dès la première décennie du XII ${ }^{\mathrm{e}} \mathrm{s}$., jusqu'en 1141.

${ }^{4}$ Ibid., vol. V, liv. IX, Oxford, 1975, p. 28.
} 
Angleterre avec Glanvill puis Bracton, de même qu'avec Jean de Salisbury, que l'influence du droit romain opère un retour en force : la trahison est, en effet, évoquée comme crime de lèsemajesté. En France, à partir du milieu du XIII ${ }^{\mathrm{e}}$ s., cette notion est devenue familière même si, estime S. Cuttler, les coutumiers font encore la part belle à la notion de trahison en tant que rupture de la fidélité 5 . Il n'est pas dans mon propos de développer plus avant ni d'évoquer les coutumiers, mais je crois qu'il est utile de se demander s'il est pertinent de penser qu'avant le milieu du XII ${ }^{\mathrm{e}}$ s., la notion de trahison, chez les auteurs médiévaux, est faiblement influencée par le droit romain. Avons-nous réellement affaire à un concept de trahison faible ? Tout en se gardant d'improbables vues téléologiques, peut-être faut-il essayer d'évaluer plus précisément différents cas de figure de la trahison et des conceptions qu'elles sous-tendent ${ }^{6}$. Pour cela, je m'appuierai principalement sur les Chroniques des comtes d'Anjou et sur l'Histoire ecclésiastique d'Orderic Vital.

J'évoquerai d'abord différents contextes où la trahison est mentionnée par les auteurs ligériens et par Orderic Vital, puis j'essaierai de mettre au jour et de préciser les héritages conceptuels des auteurs, afin de pouvoir poser un jalon dans l'étude à long terme de la trahison médiévale.

Trahir peut signifier trahir la parole donnée. Nous le voyons lorsque plusieurs seigneurs, qui avaient combattu en Angleterre aux côtés de Guillaume le Conquérant, lui font défection en retournant dans leurs terres normandes. Orderic Vital, qui rapporte l'épisode, tente cependant de justifier leurs actions en mettant en cause leurs épouses. Celles-ci, demeurées en Normandie, «brûlant de désir ardent, écrit-il, envoyaient sans arrêt à leurs maris des messagers pour les presser de revenir sans délai dans leur foyer, ajoutant que, s'ils ne le faisaient pas assez rapidement, elles prendraient de nouveaux maris ». Les malheureux époux, barons loyaux et vaillants guerriers, se retrouvent face à un dilemme : ils craignaient « d'être traîtres à la parole donnée en s'en retournant et d'être tenus pour des déserteurs. Mais d'un autre côté, que feraient-ils si leurs femmes lascives polluaient leur couche par l'adultère et portaient la honte indélébile et l'infamie à leur descendance ? » Donc, ils s'en reviennent, mais le roi confisque les terres qu'ils avaient obtenues de lui en Angleterre?

\footnotetext{
${ }^{5}$ S. H. Cuttler, The Law of Treason and Treason Trials in later Medieval France, Cambridge, 1981, p. 4-8 ; voir également D. Barthélemy, La chevalerie. De la Germanie antique à la France du XII e s., Paris, 2007, p. 468474.

${ }^{6}$ Je m'intéresserai ici à la manière dont plusieurs auteurs et rédacteurs de notices se sont emparés de notions de trahison léguées par le passé dans les domaines juridico-politique et religieux. Il va de soi qu'on n'épuisera pas la matière : en particulier, la réduction de la trahison à un comportement bestial ne sera pas développée, bien qu'elle soit essentielle, puisque, en renvoyant à des actions échappant à la nature humaine, les auteurs lui déniaient toute légitimité et justifiaient sa nécessaire répression. De la même façon, les influences des récits antiques ne seront pas évoquées ; renvoyons pour cela à la communication de D. Power dans ce volume. D'une bibliographie abondante, outre l'ouvrage de S. Cuttler, retenons principalement : D. Barthélemy, La chevalerie, op. cit., p. 85-171 ; A. Boureau, «De la félonie à la haute trahison. Un épisode : la trahison des clercs (version du XII ${ }^{\mathrm{e}}$ s.) », Le genre humain, 16-17 (1987-1988), p. 267-291 ; J. Chiffoleau, "Le crime de majesté, la politique et l'extraordinaire. Note sur les collections érudites de procès de lèse-majesté du XVII ${ }^{\mathrm{e}} \mathrm{s}$. français et sur leurs exemples médiévaux », Les procès politiques (XIV $-X V I I^{e}$ s.), actes du colloque de Rome (20-22 janvier 2003), dir. Y.-M. Bercé, Rome, 2007, p. 577-662 ; Ph. Contamine, «"Inobédience”, rébellion, trahison, lèsemajesté. Observations sur les procès politiques à la fin du Moyen Âge », Les procès politiques, op. cit., p. 63-82; J. Hudson, «Faide, vengeance et violence en Angleterre (ca 900-1200) », La vengeance, 400-1200, dir. D. Barthélemy, F. Bougard et R. Le Jan, Rome, 2006, p. 341-382.

${ }^{7}$ « Legitimi barones strenuique pugiles multipliciter anxiabantur dum regem suum cum fratribus suis et amicis sociisque sedulis circundari bellis intuebantur, ipsique si discederent infidi proditores timidique desertores palam censeri verebantur. Rursus honorabiles athletae quid facerent, si lascivae coniuges thorum suum adulterio polluerent, et progeniei suae perennis maculae notam et infamiam generarent?», Orderic Vital, op. cit., vol. II,
} 
Autre cas, plus fréquent, les châteaux et villes fortifiées étaient plus souvent enlevés grâce à la trahison qu'après des sièges parfois difficiles et incertains ${ }^{8}$. Ainsi Le Mans, où les habitants s'insurgent en 1099 contre le roi d'Angleterre, Guillaume le Roux, a été pris par trahison selon Orderic Vital ${ }^{9}$. Les habitants de cette cité sont en effet réputés avoir été changeants et infidèles; ce qu'ils ne pouvaient obtenir par la force, ils l'obtenaient par la ruse et les faux fuyants ${ }^{10}$. Versatilité, infidélité et ruse sont donc associées à la trahison.

La ruse, condamnée moralement car elle déroge aux valeurs de la chevalerie, est cependant susceptible d'entrer dans des combinaisons diverses. Nous avons vu que l'auteur de la chronique des comtes d'Anjou n'est pas allé jusqu'à attribuer la qualification de traître à Foulque le Réchin; toutefois, il ne le ménage pas vraiment. Alors que celui-ci mène des entreprises contre le seigneur d'Amboise, Sulpice, il fait des trêves captieuses avec celui-ci ${ }^{11}$ : elles lui permettent de s'en emparer par ruse et de l'emprisonner à Angers. Ici, c'est le seigneur qui est fourbe, mais le plus souvent ce sont les vassaux qui sont accusés de l'être et de trahir. Ainsi la fourberie, la tromperie et la ruse présentent-elles fréquemment une figure de la trahison quand elles servent l'infidélité. C'est à l'époque carolingienne que s'est formée la morale de la vassalité centrée sur les devoirs et la fidélité des vassaux ${ }^{12}$; c'est à la même époque que, de manière concomitante, apparaît le terme de «félonie » qui désigne spécifiquement la trahison de la fidélité due au seigneur; pour cette raison certainement, l'emploi de ce terme demeure rare chez les auteurs ecclésiastiques ${ }^{13}$.

Plutôt que de multiplier les exemples, autant mettre l'accent sur la notion de trahison chez ces auteurs afin de repérer des associations, comme nous venons déjà de le voir. La plus évidente, rappelons-le, lorsqu'on lit l'Histoire ecclésiastique, est celle qui est faite entre la trahison et la rébellion. Foulque le Réchin, lorsqu'il s'empare de son frère, se rebelle et le capture par traîtrise. De la même façon, plusieurs épisodes de l'Histoire ecclésiastique permettent de constater que leur auteur procède à cette identification lorsqu'il s'agit d'opposants au roi qui sont qualifiés comme traîtres et comme rebelles à la fois. Par exemple, le roi des Ecossais, Malcolm, «se rebella contre le roi d'Angleterre et refusa d'accomplir le service qu'il lui devait. Alors le roi Guillaume, après qu'il eût fait la paix avec son frère Robert [...], emmena ce dernier avec lui pour soumettre les traîtres qui, ayant manqué à leur foi, avaient conspiré contre le roi $^{14}$. » Même lorsque les deux termes ne sont pas associés, la

liv. 4, p. 220. En Languedoc d'une manière proche, au milieu du XII ${ }^{\mathrm{e}}$ s., le forfait, pour un fidèle qui ne respecte pas ses engagements, peut être de «trahir son serment», H. Débax, La féodalité languedocienne, $X I^{e}-X I I^{e} s$. Serments, hommages et fiefs dans le Languedoc des Trencavel, Toulouse, 2003, p. 176.

${ }^{8}$ D. Barthélemy, «Hommages, vengeances et trahisons au $\mathrm{X}^{\mathrm{e}}$ s. d'après Flodoard et Richer de Reims », $L a$ vengeance, op. cit., p. 149-158.

${ }^{9}$ «Cenomannis per proditionem subrepta est », Orderic Vital, op. cit., vol. V, liv. X, p. 254-256.

${ }^{10}$ «Cenomanni uersipelles et infidi sunt et quod fortitudine nequeunt dolis et tergiuersatione faciunt », Ibid., p. 248.

«[...] comes consilio Ambaziensium cum Supplicio dolo inducias cepit», «Gesta ambaziensium dominorum »Chroniques..., p. 91.

${ }^{12}$ Barthélemy, La chevalerie, op. cit., p. 120.

${ }^{13}$ Bien que sa première mention en latin se trouve dans une lettre de l'archevêque Hincmar de Reims de 858 adressée à Louis le Germanique, Boureau, «De la félonie », op. cit.; pour ce qui le concerne, Orderic Vital ne l'emploie pas.

14 «In illo tempore Melcoma rex Scotorum contra regem Anglorum rebellavit, debitumque seruitium ei denegavit. Porro Guillelmus rex postquam in Normannia ut supra retulimus cum Roberto fratre suo pacem fecerat, ipsumque contra infidos proditores qui contra regem conspirauerant secum duxerat », Orderic Vital, op. cit., vol. IV, liv. VIII, Oxford, 1973, p. 268 ; de la même façon, le seigneur normand Gilbert de Laigle combat des vassaux du duc insurgés à Rouen, au service de son seigneur : « grâce à son courage et à celui des siens, ainsi que grâce à l'aide des habitants de la cité qui ne soutenaient pas la trahison, il prit la porte du Sud ; joignant ses forces avec celles d'Henri [roi d'Angleterre] et des autres partisans du duc, il attaqua les rebelles dans la ville même » («Postquam Gislebertus Aquilensis tam uirtute sua suorumque quam iuuamine ciuium qui proditionis 
similitude du contexte indique nettement que les rébellions sont tenues pour des actes de trahison. Ainsi, après la mort, en 1081, de l'évêque du Mans, Arnaud, mis en place par le duc de Normandie, les habitants du Mans qui s'insurgent sont-ils caractérisés comme «toujours inobéissants et avides de rébellion contre leurs seigneurs », ce comportement étant tenu pour quasiment congénital aux habitants de cette cité dont le nom ancien dériverait de « rage canine $\gg^{15}$. Lorsque, au début du XII ${ }^{\mathrm{e}}$ s., le sire de Sablé, Robert, s'insurge contre son seigneur, le comte d'Anjou Geoffroy Plantagenêt, avec d'autres barons, Orderic Vital écrit qu'ils se rebellent ${ }^{16}$. Les occurrences de rébellions sont particulièrement nombreuses à travers 1'Histoire ecclésiastique, Orderic Vital étant l'auteur qui recourt le plus à cette notion ${ }^{17}$.

$\mathrm{Au}$ couple trahison/rébellion est également associé le terme de conspiration (conspiratio). Ainsi quand des seigneurs normands, infatués de leur importance parce qu'ils avaient acquis des richesses en Angleterre accrurent leurs ambitions, «ils firent une conspiration criminelle contre le roi $^{18}$. " L'un d'eux, par la suite, se jeta aux pieds du roi, implorant son pardon : «Nous avons conspiré contre toi et nous avons manigancé ta mort en prêtant serment ${ }^{19}$. » Aussi Orderic Vital mentionne-t-il explicitement à plusieurs reprises le crime de trahison; c'est le cas lorsque la trahison implique un meurtre. Par exemple, à l'occasion de l'assassinat de la comtesse Mabille, dame de Bellême, vers 1077-1078, un ami du meurtrier, Guillaume Pantoul, se voit imputer le crime de trahison car il en aurait été l'instigateur ${ }^{20}$. Quand la trahison atteint le roi, elle est désignée comme crime: les comploteurs normands qui projetaient d'assassiner le roi Guillaume le Roux, évoqués cidessus, «oubliant la fidélité qu'ils avaient promise à leur seigneur, se laissèrent entraîner honteusement au crime de trahison ${ }^{21}$. » Quand le comte Robert de Bellême, en guerre contre le roi Henri $\mathrm{I}^{\mathrm{er}}$, une fois vaincu, se rend, il avoue lui aussi le crime de trahison ${ }^{22}$.

Aussi devons-nous voir, afin de poursuivre ce tour d'horizon, quelles sanctions furent appliquées ou envisagées. Le roi, comme le prince, peut sanctionner, pardonner ou encore faire les deux à la fois, le pardon justifiant une atténuation ou une remise de la sanction. La

participes non erant australem portam optinuit, et Henrico aliisque ducis auxiliaribus associatus contra rebelles in urbe certamen iniit »), Ibid., p. 224 ; voir également, p. 124-126.

15 «Cenomannis a canina rabie dicta urbs est antiqua, et plebs eius finitimis est procax et sanguinolenta, dominisque suis semper contumax et rebellionis auida », Ibid., vol. II, liv. IV, p. 300.

16 «Rodbertus etiam de Sabloilo filius Lisiardi aliique proceres contra Iosfredum consulem rebellaverunt », Ibid., vol. VI, liv. XIII, p. 456.

${ }^{17}$ Alors que la trahison peut être entre tous les types de seigneurs, la rébellion ne caractérise que l'opposition au prince (roi, duc ou comte): le roi Henri $1^{\text {er }}$, après avoir vaincu le seigneur de Bellême, Robert, insurgé, lui confisqua son honor ainsi qu'aux hommes de Robert qui demeuraient en état de rébellion : « Rex itaque totum honorem Rodberti et hominum ejus, qui cum illo in rebellione perstiterant, possedit », Ibid, p. 20-30. Autres exemples : la fidélité de l'évêque du Mans, Hoël, au roi Guillaume le Conquérant provoqua contre lui la colère des meneurs de la rébellion des habitants du Mans insurgés contre le duc-roi : «Qua propter rebellionis incentores contra eum nimis irati sunt », Ibid., vol. IV, liv. VIII, p. 194. Le comte d'Anjou Geoffroy Plantagenêt, soutenant la prétention de sa femme Mathilde, fille du roi défunt Henri $\mathrm{I}^{\mathrm{er}}$, à la couronne d'Angleterre, pénètre en Normandie avec une forte armée tout en s'assurant le soutien du comte Robert de Gloucester. Cette alliance réveilla en Angleterre les espoirs d'évêques et de détenteurs de châteaux qui « dévoilèrent les mauvais desseins qu'ils couvaient secrètement en eux et se rebellèrent contre le roi » («In Anglia uero presules et oppidani quamplures ut prefatum comitem cuius potestas magna erat in utrisque regnis Andegauensibus adminiculari audierunt, nequitiam quam penes se occultabant protulerunt, et contra regem rebellauerunt »), Ibid, vol. VI, liv. XIII, p. 516). Voir aussi : Ibid., vol. V, liv. X, p. 232 ; vol. VI, liv. XI, p. 76 ; vol. II, liv.III, p. 118 ; vol. VI, liv. XIII, p. $510 \ldots$

$18 \ll[\ldots]$ in regem nefariam conspirationem fecerunt », Ibid., vol. IV, liv. VIII, p. 278.

${ }_{19}$ «Contra te conspirationem fecimus, et mortem tuam jurejurando machinati sumus », Ibid., p. 280.

${ }^{20}$ « Nam crimen ei proditionis impegabatur », Ibid., vol. III, liv. V, p. 160-162.

${ }^{21}$ « [...] fideique immemores quam domino suo promiserunt, in facinus proditionis turpiter prolapsi sunt », Ibid., vol. IV, liv. VIII, p. 278.

$22 \ll[\ldots]$ crimen proditionis confessus », Ibid., vol. VI, liv. XI, p. 30. 
sanction et le pardon ne doivent pas être opposés l'un à l'autre car la possibilité de la première rend possible le second. Revenons aux sources angevines avec une notice de l'abbaye SaintNicolas d'Angers datant vraisemblablement de 1068. Nous y apprenons qu'après l'emprisonnement de son frère, Foulque le Réchin a pris le château de Trêves, situé entre Angers et Saumur, sur la rive gauche de la Loire. Il a fait aveugler son défenseur, Hardouin, fils de Geoffroy le Fort, et il lui a confisqué ses biens patrimoniaux à cause « de son arrogance et de la défection inattendue à [sa] fidélitée ${ }^{23} »$; Foulque s'intitulant comte d'Anjou dans ce document, la sanction est donc bien relative à une trahison envers le prince. Un épisode semblable est relaté par Orderic Vital, lorsque, en 1088, le duc Robert Courteheuse entreprend une expédition dans le Maine : les Normands assiègent alors la forteresse de Saint-Céneri. Les défenseurs doivent se rendre à cause du manque de vivres ; celui qui commandait la garnison, Robert Quarrel, un fidèle de Robert de Bellême, est aveuglé sur l'ordre du duc en colère (la colère étant le comportement convenu dans une pareille situation ${ }^{24}$ ) tandis que les autres, qui avaient résisté traîtreusement, ont les membres amputés par sentence de la cour du duc ${ }^{25}$. Orderic Vital renvoie explicitement à une décision judiciaire. Observons un autre épisode de l'Histoire ecclésiastique plus riche d'enseignements. L'auteur fait dire à un noble que la loi d'Angleterre punit le traître de la décapitation et prive toute sa descendance de son juste héritage $^{26}$. Dans le même épisode, pour s'être rebellé et avoir trahi le roi, le comte de Norwich est privé de toutes ses possessions après s'être rendu et il est condamné à l'exil. Un autre noble révolté, le comte Roger, est jugé selon la loi des Normands, dit l'auteur : ses biens sont confisqués et il est condamné à la prison à vie ${ }^{27}$.

Ces épisodes sinistres m'amènent au deuxième volet de mon exposé, car ils révèlent nettement l'influence du droit romain sur la conception de la trahison, non seulement chez les auteurs mais aussi parmi les princes. Un examen plus approfondi révèle néanmoins que d'autres influences s'y ajoutent.

Les fondements juridiques de la trahison médiévale sont romains; le vocabulaire romain utilisé était perduellio, peu usité par la suite au Moyen Âge, mais c'est cependant celui qu'utilise l'auteur de l'Histoire de Geoffroy Plantagenêt pour qualifier la trahison de ses

\footnotetext{
23 «[...] omni eum haereditate paterna privaverim ob ipsius scilicet insolentiam et a mea fidelitate insperatam defectionem », Y. Labande-Mailfert, Le premier cartulaire de Saint-Nicolas d'Angers (XI'-XII s.). Essai de reconstitution précédé d'une étude historique, thèse de l'Ecole nationale des chartes, 1931, dactylographiée, corrigée par l'auteur en 1980, $\mathrm{n}^{\circ} 31$.

${ }^{24}$ B. Lemesle, «Des discours de la haine. Récits comparés de haine et de vengeances aux XI ${ }^{\mathrm{e}}$ et XII ${ }^{\mathrm{e}}$ s.», Les discours de la haine. Récits et figures de la passion dans la Cité, dir. M. Deleplace, Reims, 2009 ; S. White, "The Politics of Anger », Anger's Past. The social uses of an Emotion in the Middle Ages, dir. B. Rosenwein, Ithaca et Londres, 1998, p. 127-152.

25 «Verum deficiente alimonia castrum captum est et prefatus municeps iussu irati ducis protinus oculis privatus est. Aliis quoque pluribus qui contumaciter ibidem restiterant principi Normaniae debilitatio membrorum inflicta est ex sentencia curiae », Orderic Vital, op. cit., vol. IV, liv. VIII, p. 154. Les mutilations suscitent parfois la réprobation ouverte : ainsi le roi Henri I ${ }^{\mathrm{er}}$, après un jugement en sa cour de Rouen de chevaliers capturés à la guerre, fit-il condamner deux d'entre eux à avoir les yeux arrachés parce qu'ils s'étaient rendus coupables de parjure («pro periurii reatu oculis priuauit »); mais le comte de Flandre, Charles le Bon, osa (« audacior ») le reprocher au roi, car il est, dit-il, «inusité chez nous » («nostris ritibus inusitatem ») de punir de mutilation des chevaliers pris à la guerre alors qu'ils étaient au service de leur seigneur. A quoi le roi répondit que ce jugement était juste car ils avaient rompu la convention avec leur seigneur lige «en trahissant ignoblement leur foi » («fidemque suam nequiter prodendo legalis eri fedus disrumpere »), Ibid.., vol. VI, liv. XII, p. 352. Voir encore, ibid., vol. II, liv. IV, p. 316.

${ }^{26}$ Ibid., p. 314.

${ }^{27}$ Ibid., p. 318
} 
barons : «barones ejus [...] perduellionem meditati sunt ${ }^{28} »$. Ici, le choix du terme s'explique parce que l'auteur truffait son texte d'emprunts aux auteurs latins classiques. Sinon, les autres auteurs, comme Orderic Vital, usent couramment de «proditio », «proditor ». Les caractères de la trahison passés en revue se trouvent dans la lex julia maiestatis reprise dans le livre 48 du Digeste. La «perduellio » était, sous l'empire, associée à la lèse-majesté et S. Cuttler a montré que c'était ce corpus juridique qui allait servir de fondement à la loi de trahison à la fin du Moyen $\mathrm{A} g e^{29}$, quoique, en réalité, contrairement à ce que le titre de son ouvrage suggère, il n'a pas existé en France de loi de trahison comme ce fut le cas en Angleterre. Cependant, il est intéressant de remarquer que, avant le milieu du XII ${ }^{\mathrm{e}}$ s., à une époque où mes documents n'associent pas explicitement la trahison contre le roi à la lèse-majesté, ils reprennent tous les autres caractères de la loi romaine de trahison, qu'il s'agisse du roi ou du prince territorial. La «proditio» figurait dans le Digeste où elle désignait, plus spécifiquement que la «perduellio », la livraison à l'ennemi d'une place forte. Plus largement, parmi les crimes décrits dans le Digeste comme relevant de la trahison, se trouve la sédition, sous sa forme armée ou autrement, le fait d'apporter une aide matérielle ou financière à l'ennemi, la désertion ou la défection. Nous reconnaissons dans cet extrait les situations décrites plus haut. Le châtiment des traîtres, quant à lui, est exposé dans la lex Quisquis, une loi impériale promulguée en 397 et reprise dans le Code théodosien. Elle stipule que le traitre devait être exécuté et que ses propriétés devaient être confisquées. Les héritiers étaient privés de la succession ${ }^{30}$. Nous avons vu que ce sont ces dispositions qu'Orderic Vital attribue à la loi anglaise.

Indépendamment du fait que les dispositions ont été variées, le vocabulaire employé par le moine chroniqueur normand révèle aussi la marque de plusieurs auteurs chrétiens des premiers siècles et au-delà. Ainsi, la mort de l'évêque du Mans, Arnaud, provoque-t-elle une sédition des habitants de la cité qu'Orderic Vital décrit comme «semper contumax et rebellionis avida ${ }^{31} »$. Dans un autre épisode que j'ai évoqué plus haut, le comte Roger, qui est emprisonné à cause de sa trahison, continue à offenser le roi : «contumacibus actis implacabiliter regem offendit ${ }^{32} »$. Troisième cas : après que le duc Robert Courteheuse a pris la place fortifiée de Saint-Céneri, ceux qui avaient résisté traîtreusement ont les membres amputés : «aliis quoque pluribus qui contumaciter ibidem restiterant principi Normanniae ${ }^{33}$ ». C'est bien le terme «contumax » qui revient, associé à la trahison. Ce terme existe dans le droit romain où il désigne le refus de répondre à une citation, donc au fait de ne pas obtempérer à un ordre donné par un magistrat. Un passage du code théodosien va plus loin en ce qu'il associe la contumace à la rébellion ${ }^{34}$, le terme servant à qualifier les rebelles qui troublent la paix publique. Mais surtout les auteurs chrétiens des premiers siècles ont souvent usé des termes de «contumax » ou «contumacia» et de « rebellio » ou « rebelles », parfois en les associant, à l'instar du code théodosien. C'est le cas de saint Jérôme (347-420), de Jean Cassien (360-435), ainsi que des papes Léon $1^{\mathrm{er}}(440-461)$ et Gélase $1^{\mathrm{er}}(492-496)^{35}$, et de la

\footnotetext{
${ }^{28}$ «Historia Gaufredi ducis Normannorum et comitis Andegavorum », Chroniques, op. cit., p. 201.

${ }^{29}$ Cuttler, The Law, op. cit, p. 7.

${ }^{30}$ Ibid., p. 6-7.

${ }^{31}$ Orderic Vital, op. cit., vol. II, liv. IV, p. 300.

${ }^{32}$ Ibid., p. 318.

${ }^{33}$ Ibid., vol. IV, liv. VIII, p. 154.

34 «Nec enim eos fas est adimi debitae se veritati, qui pacem publicam actuum perturbatione confusam rebelli contumacia miscuerunt »(C. Th. XI, 36, 31).

35 « Nam eumdem contumacem populum et rebellem » (saint Jérôme, « Commentarii in librum Job », dans J. P. Migne, Patrologia latina, vol. 26, col. 769) ; « sed rebellionis spiritu contumaciam nos eidem noverimus offere » (Jean Cassien, De coenobium institutis, ibid., vol. 49, col. 342) ; « nec contumaces atque rebelles acquievissent » (Léon $1^{\mathrm{er}}$, ibid. vol. 55, col. 1105) ; «contumaciter et contra constitutum rebelles ad comitatum filii mei regis putaverunt esse properandum» (Gélase $1^{\mathrm{er}}$, ibid., vol. 59, col. 99).
} 
règle de saint Benoît, au chapitre $62^{36}$. Chez ces auteurs, «contumacia » désigne presque toujours le refus d'obéir à la loi de Dieu, ou au roi; la règle de saint Benoît, quant à elle, l'oppose au devoir d'obéissance du moine ${ }^{37}$.

Qu'en est-il enfin, plus largement, des termes « rebellio» et « rebellare»dans les textes juridiques et chez les auteurs chrétiens avant l'époque d'Orderic Vital ? Leur emploi est très secondaire dans le droit romain et au Moyen Âge : il faut attendre le $\mathrm{XIV}^{\mathrm{e}}$ s. pour que la rébellion devienne une qualification juridique. Le terme est également peu utilisé dans la littérature latine classique, mais relativement fréquemment chez les auteurs chrétiens ainsi qu'on le vérifie à l'aide de l'édition électronique de la Patrologie latine. Au cours du Moyen Âge, nous le trouvons employé chez les annalistes ainsi que chez les auteurs de chroniques ou de gesta. $\mathrm{Au} \mathrm{XI}$ s., les papes, dans leur correspondance, et Pierre Damien (qui associe, lui aussi, « rebelles » et «contumaces » ${ }^{38}$ ), l'emploient relativement peu, de même qu'Yves de Chartres. En revanche, Guillaume de Poitiers dans son Histoire de Guillaume le Conquérant l'utilise plusieurs fois dans des contextes identiques à ceux que nous avons rencontrés chez Orderic Vital, qui est vraiment le champion pour son emploi. Le mot est évidemment plus polémique que juridique à cette époque mais il est bien relatif au crime de trahison, à la gravité de la faute par conséquent.

Pour conclure, revenons à notre interrogation initiale : y a-t-il eu un concept faible de la trahison avant le milieu du XII ${ }^{\mathrm{e}}$ s. ? Est-elle réduite à l'infidélité féodale, à la félonie (un terme que les auteurs que j'étudie n'emploient pas) ? Un inventaire même non exhaustif des différents types de situations et de documents permet de répondre par la négative à cette question. On voit, au contraire, que la notion romaine de trahison, avec les châtiments qui la punissent, marque fortement les auteurs, mais aussi les acteurs des $\mathrm{XI}^{\mathrm{e}}$ et $\mathrm{XII}^{\mathrm{e}}$ s. On voit de même qu'ils ont enrichi le concept juridique romain avec les apports des auteurs ecclésiastiques relativement au refus d'obéissance et avec l'utilisation de la notion de rébellion léguée par nombre d'auteurs au cours des siècles précédents. La trahison, sans être qualifiée juridiquement, est tenue pour un crime lorsqu'elle est commise contre le roi ou contre le prince.

C'est pourquoi ce qui fait sens dans les récits que j'ai cités est l'existence de normes connues qui fournissent des références aux princes et aux auteurs ecclésiastiques; on ne décèle donc aucun archaïsme dans tout cela car elles ont une utilité politique. Leur application peut naturellement se révéler pragmatique et répondre à des situations concrètes différentes. Ainsi, le pardon, tout comme les mutilations féroces, l'emprisonnement, l'exil et la confiscation se lisent dans les récits et peuvent être combinés pour aboutir à toutes sortes de mitigations et atténuations. La possibilité même de la sanction la plus forte, à laquelle correspond le vocabulaire de la trahison et de la rébellion employé par les auteurs pour qualifier les actes d'insoumission des nobles vis-à-vis du prince, est ce qui nous importe le plus : elle est la marque du pouvoir politique princier et royal, comme le pardon qui en est l'alternative obligée, quand le prince peut manifester son indulgence et sa bonté, déployer la disposition de son âme à épargner ou à adoucir la rigueur impitoyable de la peine. Le vocabulaire de la trahison et de la rébellion n'est pas non plus simple surenchère dans une sorte de jeu de pouvoirs où s'exercent des rapports de force, ni un artifice que les auteurs

\footnotetext{
${ }^{36}$ La Règle de saint Benoît, éd. et trad. A. de Vogüe, Paris, Cerf, 1972, p. 642. Mon attention sur les occurrences de ce mot dans la règle de saint Benoît a été attirée au séminaire que Jacques Chiffoleau lui a consacré en 2007 et 2008 à l'École des Hautes Études en sciences sociales; j'en profite pour l'en remercier.

37 «culpa contumaciae reatui deputetur » (La Règle du Maître, éd. A. de Vogüe, t. 1, Paris, Cerf, 1964, p. 386).

${ }^{38}$ J.P. Migne, Patrologia latina, vol. 144, col. 211 et 770.
} 
utiliseraient pour mieux souligner la clémence des princes en réponse aux actes hautement reprochables des nobles révoltés. Certes, les rapports de force déterminent la conduite effective du prince et, bien souvent, sont l'explication au pardon accordé et à la réduction des sanctions dont les traîtres sont passibles. Mais les rapports de force n'expliquent que chaque situation considérée dans sa singularité. Les références aux sanctions graves qui punissent le crime de trahison envers le prince relèvent d'autres enjeux : elles sont l'une des formes possibles du pouvoir en œuvre et elles sont adossées au droit romain dont les principes, sur ce plan, n'ont jamais été oubliés en dépit d'une apparente mise en sommeil ; en elles résonne aussi le vocabulaire des auteurs chrétiens de l'Antiquité tardive et du très Haut Moyen Âge dans ce qui se rapporte à la soumission voulue de la noblesse vis-à-vis du prince.

Au total, on ne peut pas se satisfaire de l'idée d'une conception faible de la trahison ; elle n'entre pas encore dans une construction institutionnelle, mais elle contient néanmoins déjà les éléments qui serviront à une telle construction. Ceci nous permet de comprendre pourquoi, dès la seconde moitié du $\mathrm{XII}^{\mathrm{e}}$ s., des auteurs ont pu procéder à une réflexion juridique construite de la trahison en associant celle qui atteint le souverain au crime de lèsemajesté. 